\title{
Determinação da despesa com energia elétrica na lavagem de filtro rápido de estação de tratamento de água
}

\author{
Electricity cost of rapid filter backwashing in a water treatment plant

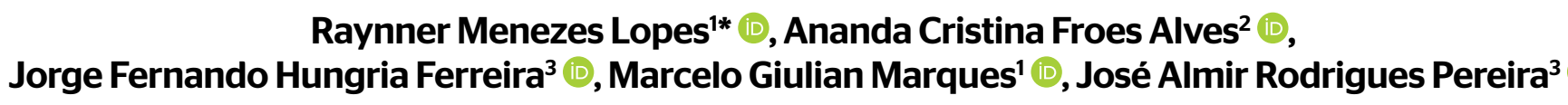

$\square$

\begin{abstract}
RESUMO
O estudo teve por objetivo determinar a despesa com energia elétrica na lavagem de filtro rápido de uma estação de tratamento deágua(ETA), cuja vazão é de 45 L/s. Para isso, foi realizado monitoramento hidroenergético para a análise do desempenho no bombeamento de água de lavagem. Adicionalmente, foi monitorada a qualidade da água do efluente de três lavagens no filtro 4 e realizado um levantamento das informações tarifárias de energia elétrica da unidade de tratamento. Com os resultados obtidos, observou-se que os valores de turbidez do efluente, ao final das três lavagens, no caso, 31, 30 e 27 UNT, não atingiram os limites mínimos recomendados na literatura técnica, que é de, pelo menos, 15 UNT. Foi observada também a dificuldade de prolongamento do tempo de lavagem, a fim de alcançar o padrão da literatura, pois o reservatório elevado (REL) de água de lavagem (água tratada), também alimenta a rede de distribuição, e poderia esvaziar completamente. Levando em conta essas limitações e a qualidade final do efluente de lavagem, observou-se que as lavagens devem ser finalizadas aos 380 segundos (6,3 minutos), tempo que consome um volume de 23,36 mªlavagem. A despesa de água de lavagem foi calculada em 1,36 R\$/m33, o que resultou no valor de 31,83 R\$/lavagem. Para as lavagens de todos os filtros da estação, as despesas foram de 254,64 R\$/dia, 7.639,20 R\$/mês e 91.670,4 R\$/ano. Esses valores são considerados elevados para o sistema de tratamento estudado.
\end{abstract}

Palavras-chave: energia elétrica; lavagem de filtros; indicadores; desempenho hidroenergético.

\begin{abstract}
This paper aims to determine the cost of electricity for rapid filter backwashing procedures at water treatment plants, whose flow rate is $45 \mathrm{~L} / \mathrm{s}$. Flow and electricity consumption were monitored in order to analyze the performance of the pumping systems. Additionally, the effluent water quality of three washes was monitored in filter 4 and a survey of the electricity fare data of the treatment unit was carried out. With the results obtained, it was observed that the effluent turbidity values at the end of the three washes, in this case 31, 30, and 27 NTU, did not reach the minimum values recommended in the technical literature, which is at least 15 NTU. It was also observed the impossibility of prolonging the backwashing time in order to reach the standard of the literature, due to the double function of the elevated washing water reservoir (treated water), whose main purpose is to provide treated water to the water distribution network. Taking into account these limitations and the final quality of the wash effluent, it was observed that the duration of backwashing procedures should be around 380 seconds (6.3 minutes), consuming $23.36 \mathrm{~m}^{3} /$ wash. The backwashing procedure cost was estimated at $\mathrm{R} \$ 1.36 / \mathrm{m}^{3}$, which resulted in $\mathrm{R} \$ 31.83 /$ wash. Considering the whole filtration unit, the backwashing procedure cost was R $\$ 254.64 /$ day, R\$ 7,639.20/month, and $\mathrm{R} \$ 91,670.4 /$ year. This value can be classified as too expensive considering the treatment plant studied.
\end{abstract}

Keywords: electrical power; filter backwashing; indicators; hydraulic and energetic performance

\section{INTRODUÇÃO}

A diminuição dos níveis de água nos mananciais e o aumento do consumo de energia elétrica afetam significativamente o desempenho do setor produtivo brasileiro, exigindo ações do poder público para minimizar os riscos ambientais e as despesas relacionadas ao fornecimento de água e de energia elétrica.

Essa situação é marcante no setor de saneamento básico, tendo em vista que, para atender ao volume de água demandado nas cidades,

$\square$

'Universidade Federal do Rio Grande do Sul - Porto Alegre (RS), Brasil.

¿Universidade de São Paulo - São Paulo (SP), Brasil.

3Universidade Federal do Pará - Belém (PA), Brasil.

*Autor correspondente: raynner.lopes@ufrgs.br

Recebido: 27/O2/2017 - Aceito: 12/02/2019 - Reg. ABES: 176367 
existe a necessidade de se utilizar mananciais cada vez mais distantes, naturalmente aumentando o consumo e a despesa de energia elétrica.

De acordo com Brasil (2018), no ano de 2016, último ano computado no Sistema Nacional de Informações sobre Saneamento (SNIS), foi registrado consumo total de energia elétrica de 10.6 TWh nos Sistemas de Abastecimento de Água (SAAs) do Brasil.

No período de um ano, as despesas com energia elétrica nos setores de abastecimento de água e de esgotamento sanitário do Brasil aumentaram 5,48\% (aproximadamente R\$ 281,7 milhões), passando de R\$5.136,24 milhões, no ano de 2015, (BRASIL, 2017) para R\$ 5.417,9 milhões, em 2016 (BRASIL, 2018).

É importante observar que o maior consumo de energia elétrica ocorre no setor de abastecimento de água e que, progressivamente, aumenta a participação da energia elétrica nas despesas de exploração (DEX) dos prestadores de serviços de saneamento básico, sendo que a defasagem de dois anos para a publicação dessas informações no SNIS impossibilita a tomada de imediata de decisões para reduzir esse consumo.

Pereira e Condurú (2014) observam que a DEX está relacionada ao custo operacional na obtenção, no processamento e na distribuição de água, precisando ser verificada em todas as unidades, pelo fato de impactar diretamente na qualidade do serviço prestado e no valor da tarifa cobrada ao usuário.

Entre os fatores relacionados ao aumento na despesa de energia elétrica, está o consumo de energia no horário de ponta $(\mathrm{P})$ elétrico, que é definido pela concessionária de energia elétrica como o período de 3 horas consecutivas, em que o valor do kWh é mais elevado (BRASIL, 2010), podendo chegar a 8 vezes o valor normal da tarifa, dependendo da modalidade tarifária de enquadramento do sistema.

No atual cenário de aumento das despesas e de dificuldade para a aprovação de ajustes tarifários, os prestadores de serviço de saneamento básico precisam se atentar para a Resolução $n^{\circ} 1.859$, de 27 de fevereiro de 2015, da Agência Nacional de Energia Elétrica (ANEEL), pois as modificações no sistema tarifário, incluindo valores diferenciados por bandeiras tarifárias, resultam em aumento da despesa de energia elétrica na operação dos SAAs. Esse fato é comprovado pelo aumento do percentual de despesa com energia elétrica na DEX, que, entre os anos de 2013 e 2014, foi de $0,38 \%$ e, entre os anos de 2014 e 2015 (ano de publicação da Resolução n 1.859 da ANEEL), subiu para 4,37\%. Apesar do aumento nas despesas de energia elétrica, entre os anos de 2014 e 2015, houve redução no consumo de energia elétrica nos SAAs, sendo esse consumo, no ano de 2014, de 11.573.276,58 kWh, 5,26\% acima do consumo de energia elétrica no ano de 2015 (BRASIL, 2015; 2016; 2017). Contudo, mesmo com a diminuição do consumo de energia elétrica, as despesas aumentaram, evidenciando a importância que deve ser atribuída à gestão das despesas de energia elétrica.
Nesse cenário, o pequeno controle dificulta a sustentabilidade econômico-financeira de muitos prestadores de serviços, principalmente com as falhas gerenciais ou operacionais aumentando o consumo e as despesas de energia elétrica nos SAAs. Com isso, a gestão hidroenergética das instalações é necessidade urgente e precisa de soluções para atender às demandas de água com a menor despesa de energia elétrica.

Apesar do desenvolvimento de importantes estudos de eficiência hidroenergética em SAAs, como os métodos de programação computacional propostos por Firmino et al. (2006), Cheung et al. (2003) e Gumier e Luvizotto Junior (2007) para a redução do consumo de energia elétrica e de perdas de água em unidades de bombeamento e distribuição, ainda é escasso o número de pesquisas relacionadas ao controle hidroenergético em estações de tratamento de água (ETAs).

Considerando-se a diferença entre os volumes de água na entrada e na saída da ETA, é preciso avaliar a quantidade que recebe energia elétrica no volume de água perdido ou utilizado na limpeza das instalações. De acordo com Brasil (2004), esse volume de água em ETAs brasileiras pode variar de 2 a 10\% do volume total de água tratada.

Freitas et al. (2010) observam que a água utilizada para a retrolavagem de filtros rápidos pode apresentar cerca de $5 \%$ (ou mais) do volume de água tratada. Esses valores indicam a necessidade de os procedimentos hidroenergéticos serem aprimorados na rotina operacional das ETAs.

Assim, o objetivo do presente estudo foi realizar uma análise do desempenho hidroenergético para propor ações de redução do consumo e da despesa de energia elétrica na operação de lavagem de filtro rápido de ETA do tipo desferrização.

\section{MATERIAIS E MÉTODOS}

O estudo foi realizado na ETA do SAA da Cidade Universitária Prof. José da Silveira Netto, campus principal da Universidade Federal do Pará (UFPA), localizado no município de Belém, Estado do Pará. Esse SAA é constituído por unidades de captação, adução, tratamento, elevação, reservação e distribuição de água, fornecendo água potável para os setores básico, profissional, de saúde e de educação física do campus da UFPA, conforme representado na Figura 1.

A ETA é do tipo desferrização, tendo dois módulos de tratamento da vazão total de $45 \mathrm{~L} / \mathrm{s}$. Cada módulo é constituído de um aerador do tipo tabuleiro, dois leitos de contato e dois filtros rápidos por gravidade, de fluxo descendente, além da unidade de desinfecção por cloração. Assim, existem quatro filtros na unidade de tratamento pelos quais são removidas partículas suspensas, com destaque para o ferro precipitado após a aeração. A acumulação desse material no leito filtrante requer a paralisação da carreira de filtração, para a manutenção da unidade de filtração. 
A lavagem de cada filtro é realizada em fluxo ascensional (contracorrente), em intervalo entre 12 e 18 horas. Para isso, o volume de água empregado na lavagem é bombeado para reservatório elevado (REL) de $120 \mathrm{~m}^{3}$, com cota de fundo de 12,6 m, que também é utilizado para o abastecimento de água nos setores do campus. Essa dupla função do REL resulta em lavagens dos filtros em horários não programados,

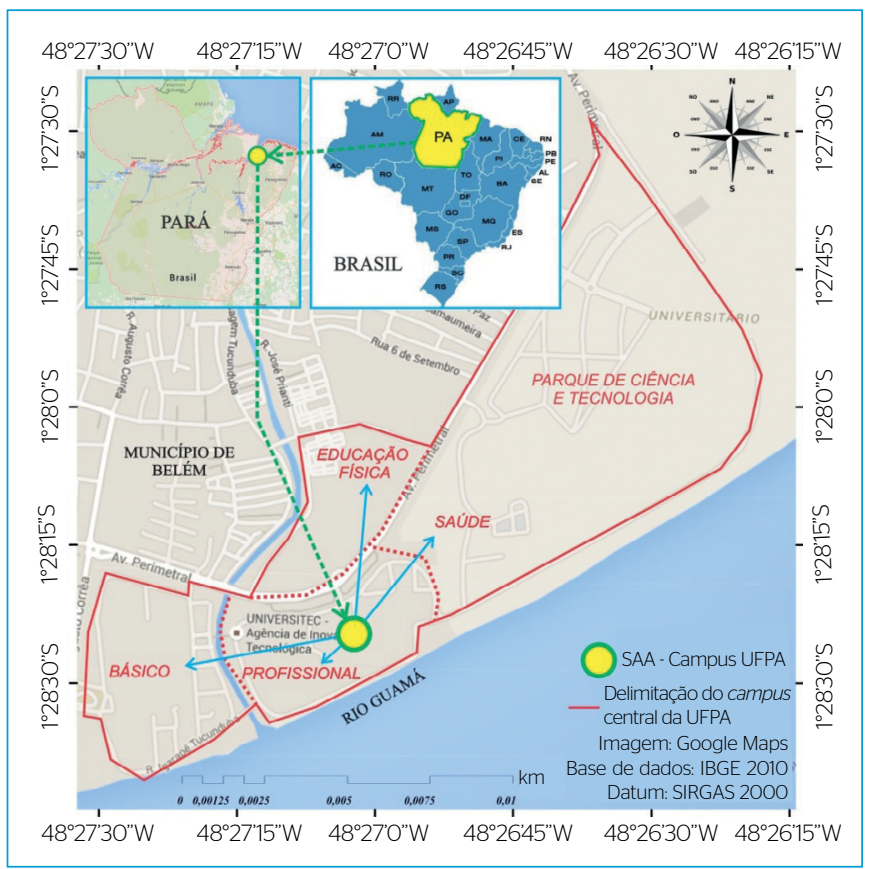

Figura 1 - Mapa de localização do sistema de abastecimento de água do campus universitário da Universidade Federal do Pará. como no P, aumentando significativamente a despesa de energia elétrica no bombeamento da água. Além disso, a falta de equipamentos de medição e controle dificulta a padronização das ações operacionais para atingir a eficiência hidroenergética.

\section{Delineamento experimental}

A pesquisa foi realizada no mês de outubro de 2016, sendo dividida nas três etapas representadas na Figura 2.

$\mathrm{Na}$ etapa 1 foi monitorada a qualidade da água de três lavagens consecutivas de um único filtro (no caso, o filtro 4). O uso de um único e exclusivo filtro se deu por duas razões: a primeira por recomendação dos gestores da unidade, pois o sistema já estava com um de seus filtros inoperantes e o filtro 4 necessitou de ajuste na operação, para viabilizar o monitoramento; e a segunda por ter sido necessário realizar o monitoramento do tempo de carreira de filtração, para verificar a influência dessa carreira nas suas respectivas lavagens.

Tanto os tempos de carreira quanto os de lavagem foram definidos em consonância com os operadores do sistema, para que não houvesse falta de água no reservatório, que também abastece o campus universitário. Assim, houve carreiras de filtração que tiveram de "aguardar" o reservatório estar com água suficiente para lavar o filtro sem que o abastecimento fosse interrompido. Da mesma forma, as lavagens foram estendidas ao máximo possível, considerando-se as limitações do REL.

Para o tempo da primeira carreira de filtração não houve monitoramento, uma vez que o tempo da primeira lavagem, por razões operacionais, foi o padrão normalmente adotado pelo operador da ETA, que é de 6,8 minutos. Assim, não foi considerada a influência da

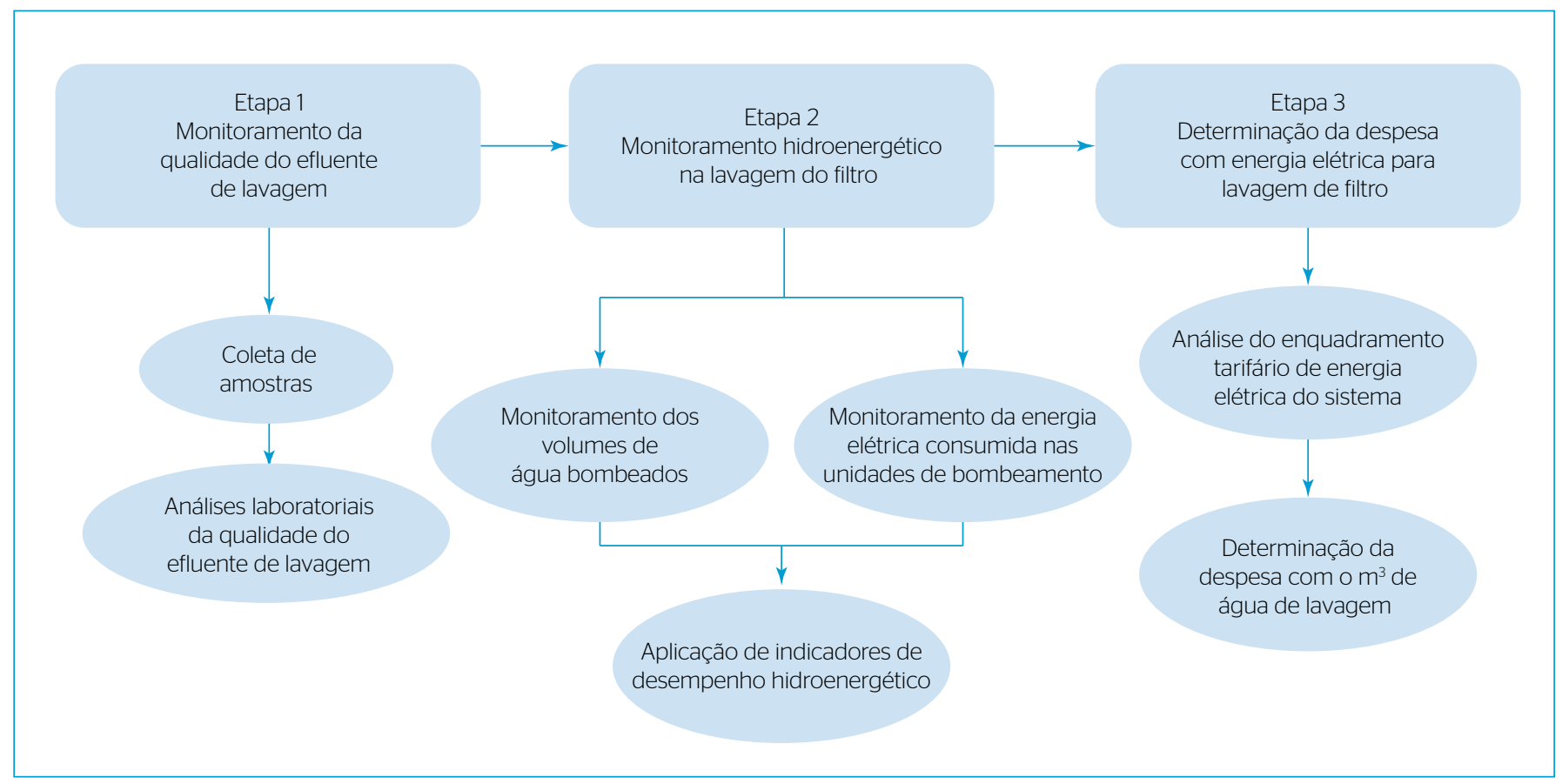

Figura 2 - Etapas executadas na pesquisa. 
carreira nessa lavagem. Após 15 horas de filtração (carreira) foi realizado o monitoramento da segunda lavagem, com duração de 9 minutos. Depois de 11 horas de filtração, foi monitorada a terceira lavagem, com duração de 10 minutos.

Nessa etapa foram coletadas amostras para monitoramento dos parâmetros turbidez e sólidos suspensos totais (SSTs) nas três operações de lavagem do filtro. Para isso, foram utilizados frascos de $300 \mathrm{~mL}$, sendo as coletas realizadas em intervalos de 20 segundos, totalizando 70 amostras coletadas. As amostras foram analisadas no Laboratório de Química Ambiental da Universidade Federal Rural da Amazônia (UFRA), sendo os valores de turbidez e SST obtidos no equipamento Hach Colorimeter DR 890, com medição conforme o Absorptmetric Method.

$\mathrm{Na}$ etapa 2 foram monitoradas as variáveis hidroenergéticas relacionadas com as lavagens dos filtros, no caso o volume e o consumo de energia na elevatória de água bruta (EAB), o volume e o consumo de energia na elevatória de água tratada (EAT) e o volume de água de lavagem dos filtros. Para isso, foram instalados equipamentos de medição em cinco pontos de monitoramento.
$\mathrm{O}$ volume de água bruta foi monitorado no ponto V1, instalado na linha de recalque de água bruta, entre o poço de captação e o filtro, sendo a energia elétrica utilizada no bombeamento dessa água monitorada no quadro de comando do conjunto elevatório do poço de captação (ponto E1).

$\mathrm{O}$ volume de água tratada foi monitorado no ponto $\mathrm{V} 2$, instalado na linha de recalque de água tratada, entre a EAT e o REL. Por sua vez, a energia elétrica empregada nesse volume de água foi monitorada no quadro de comando dessa unidade de bombeamento (ponto E2).

O monitoramento do volume de água de lavagem foi realizado no ponto V3, localizado na tubulação entre o REL e o filtro, conforme pode ser observado na Figura 3.

Os volumes de água foram monitorados em equipamentos de medição de vazão, modelo UF 801 - P, com precisão de +/- 0,5\% FS, portáteis e não intrusivos (clamp on), ultrassônicos e com atuação por tempo de trânsito. Por sua vez, o monitoramento da energia elétrica consumida foi realizado em analisadores de qualidade de energia, modelo RE6000, com precisão de $0,2 \%$. O monitoramento de volume de água e de energia elétrica foi realizado conforme observado na Tabela 1.

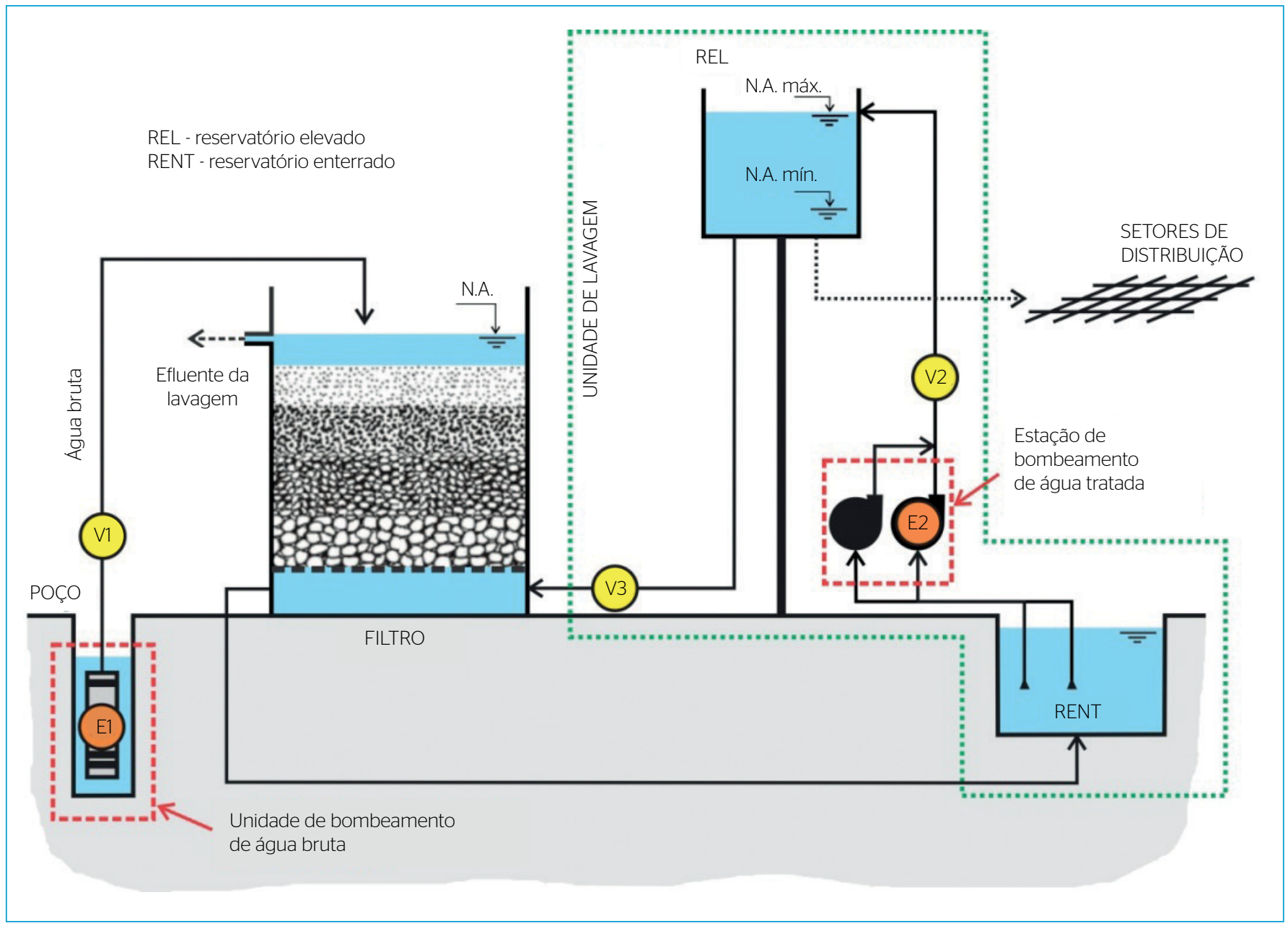

Figura 3 - Pontos de monitoramento de volume de água e energia elétrica no sistema. 
Os dados hidráulicos e elétricos obtidos no monitoramento foram utilizados na determinação do índice de perdas, do consumo específico de energia elétrica (CEE) e do consumo específico de energia normalizado (CEEN).

Apesar de comumente ser aplicada para a unidade de distribuição do SAA, a expressão matemática do índice de perdas pode ser utilizada para relacionar o volume de água produzido na ETA e o volume utilizado na lavagem dos filtros. Para facilitar o entendimento no presente trabalho, esse indicador foi expresso como índice de perda de água na lavagem dos filtros (IPALF), conforme apresentado na Equação 1:

$\mathrm{IPA}_{\mathrm{LF}}=\frac{V_{P}-V_{U}}{V_{P}} \cdot 100 \%$

Em que:

$\mathrm{V}_{\mathrm{p}}=\mathrm{o}$ volume em $\mathrm{m}^{3}$ de água tratada produzida na ETA (que, neste estudo, equivale aos volumes obtidos no ponto V1);

$\mathrm{V}_{\mathrm{U}}=\mathrm{o}$ volume em $\mathrm{m}^{3}$ de água de lavagem dos filtros (equivalente aos volumes monitorados no ponto V3 deste trabalho).

O CEE, expresso em $\mathrm{kWh} / \mathrm{m}^{3}$, é um indicador definido como a relação entre a energia consumida na unidade de bombeamento $\left(C_{E}\right)$, expressa em $\mathrm{kWh}$, e o volume de água bombeado $\left(\mathrm{V}_{\mathrm{B}}\right), \mathrm{em} \mathrm{m}^{3}$, sendo representado na Equação 2:

$\mathrm{CEE}=\frac{C_{E}}{V_{B}}$

O indicador CEE foi utilizado para determinar o consumo de energia elétrica por $\mathrm{m}^{3}$ de água bombeada tanto na EAB quanto na EAT, para, posteriormente, agregar os consumos de energia elétrica no $\mathrm{m}^{3}$ de água transportada na ETA, conforme recomendação de Pereira e Condurú (2014). Essa diretriz foi considerada, em razão de o volume de água de lavagem ter sido bombeado duas vezes na ETA, incorporando, portanto, dois consumos de energia elétrica (EAB + EAT).

A aplicação do CEEN, expresso em $\mathrm{kWh} / \mathrm{m}^{3} / 100 \mathrm{~m}$, foi realizada para análise comparativa do desempenho entre as unidades de bombeamento do sistema (EAB e EAT). De acordo com Alegre et al. (2004),

Tabela 1 - Informações detalhadas do monitoramento hidroenergético.

\begin{tabular}{l|c|c|c|c}
$\begin{array}{l}\text { Monito- } \\
\text { ramento }\end{array}$ & Pontos & Periodo & $\begin{array}{c}\text { Intervalo entre } \\
\text { as aquisições (s) }\end{array}$ & $\begin{array}{c}\text { Número de da- } \\
\text { dos aquisitados }\end{array}$ \\
\cline { 2 - 5 } \multirow{2}{*}{ EAB } & V1 & 48 horas & 10 & 17.280 \\
\cline { 2 - 5 } & E1 & 48 horas & 10 & 17.280 \\
\hline \multirow{2}{*}{ EAT } & V2 & 48 horas & 10 & 17.280 \\
\cline { 2 - 5 } & E2 & 48 horas & 10 & 17.280 \\
\hline $\begin{array}{l}\text { Água de } \\
\text { lavagem }\end{array}$ & V3 & 25,8 minutos & 10 & 134 \\
\hline Total & & & 69.254 \\
\hline
\end{tabular}

EAB: elevatória de água bruta; EAT: elevatória de água tratada. esse índice é definido como a quantidade média de energia gasta $\left(C_{E}\right)$ em $\mathrm{kWh}$ para uma unidade de bombeamento elevar $1 \mathrm{~m}^{3}$ de água a $100 \mathrm{~m}$ de altura manométrica $(\mathrm{Hm})$, conforme apresentado na Equação 3 :

$$
\mathrm{CEEN}=\frac{C_{E}}{V_{B}\left(\frac{\mathrm{HM}}{100}\right)}
$$

Os valores de alturas manométricas utilizados no cálculo do CEEN foram obtidos para os volumes de água monitorados nos pontos $\mathrm{V} 1 \mathrm{e}$ V2 das tubulações de recalque da EAB e da EAT.

Na etapa 3 foi determinada a despesa com energia elétrica nas unidades de bombeamento (EAB e EAT), identificando a classe tarifária na qual o sistema está enquadrado, bem como o $\mathrm{P}$ e o FP, a tarifa do kWh consumido, a tarifa de demanda contratada e a tarifa de energia reativa excedente consumida. Esses parâmetros são apresentados na Equação 4, utilizada para a obtenção do valor da despesa final com energia elétrica de uma unidade consumidora qualquer, independentemente da classe tarifária, correspondente a um período $p$ de leitura, também chamado ciclo de faturamento (GOMES, 2009).

$\mathrm{DT}=[(\mathrm{FC}+\mathrm{FD}+\mathrm{FER}+\mathrm{FDR}) \mathrm{x}(1-\mathrm{desc})+\mathrm{FU}] \mathrm{x}\left(\frac{1}{1-\mathrm{icms}}\right)+$ outros

Em que:

$\mathrm{DT}=\mathrm{a}$ despesa total com energia elétrica, em R\$;

$\mathrm{FC}=\mathrm{o}$ valor total do faturamento do consumo de energia ativa $(\mathrm{kWh})$, em R\$;

$\mathrm{FD}=\mathrm{o}$ valor total do faturamento da demanda de potência ativa (kW), em R\$;

$\mathrm{FER}=\mathrm{o}$ valor total do faturamento do consumo de energia reativa (kVArh) excedente, em R\$;

$\mathrm{FDR}=\mathrm{o}$ valor total do faturamento da demanda de potência reativa (kVAr) excedente, em R\$;

$\mathrm{FU}=\mathrm{o}$ valor total do faturamento da demanda de ultrapassagem de potência ativa $(\mathrm{kW})$, em $\mathrm{R} \$$;

desc $=$ a expressão decimal do desconto percentual incidente sobre as tarifas e aplicáveis aos fornecimentos de energia a unidades consumidoras de classes/grupos específicos (consumidores rurais, serviço público, etc.) descritos na legislação vigente;

icms $=$ a expressão decimal da alíquota do ICMS, aplicável a classes específicas de consumo descritas na legislação.

Como o objetivo da pesquisa foi a determinação da despesa de energia elétrica na operação, não foram consideradas as parcelas referentes aos descontos e tributos a reter, como ICMS, PIS e COFINS, CLSS, entre outros, uma vez que são variáveis mensalmente e de acordo com cada estado. Desse modo, foi utilizada a Equação 4: 
Os valores de DT foram determinados para as operações diária, mensal e anual.

\section{RESULTADOS E DISCUSSÃO}

No monitoramento da qualidade do efluente de lavagem dos filtros foi constatada grande variação nos valores de turbidez e de SST nos três períodos de lavagem. Foram registrados valores máximos de turbidez e de SST no efluente da lavagem de 4400 UNT e 3.000 mg.L ${ }^{-1}$ na primeira lavagem, de $5800 \mathrm{UNT}$ e $3.700 \mathrm{mg} . \mathrm{L}^{-1}$ na segunda lavagem e de 4000 UNT e $2.600 \mathrm{mg} . \mathrm{L}^{-1}$ na terceira lavagem.

Esses valores foram diminuindo no decorrer das lavagens, sendo registrados, na última amostra do efluente, valores de $31 \mathrm{UNT}$ e 19 mg.L.- ${ }^{-1}$; de 30 UNT e 20 mg.. $L^{-1}$; e de 27 UNT e 17 mg.L.-1 na primeira, segunda e terceira lavagens, respectivamente. Na Figura 4 podem ser observados os perfis do decaimento dos valores de turbidez e de SST das três lavagens no filtro 4 .

Cleasby e Logsdon (1999) citam que a interrupção da lavagem do filtro deve ocorrer quando a qualidade do efluente alcançar 10 UNT. Por sua vez, Kawamura (2000) estabeleceu a faixa entre 10 e 15 UNT para cessar a lavagem. Considerando-se as recomendações desses autores, as lavagens do filtro 4 monitorado deveriam ter períodos maiores de duração, já que os valores de turbidez das últimas coletas não atingiram os valores recomendados.

Além do tempo de lavagem, o monitoramento dos parâmetros turbidez e SST do efluente são fundamentais para a limpeza eficiente e com despesa reduzida dos filtros. Altas concentrações de partículas no efluente de lavagem normalmente estão acima da faixa de leitura de equipamentos simples, o que aumenta o erro ao requerer a diluição das amostras e a correção proporcional do resultado.

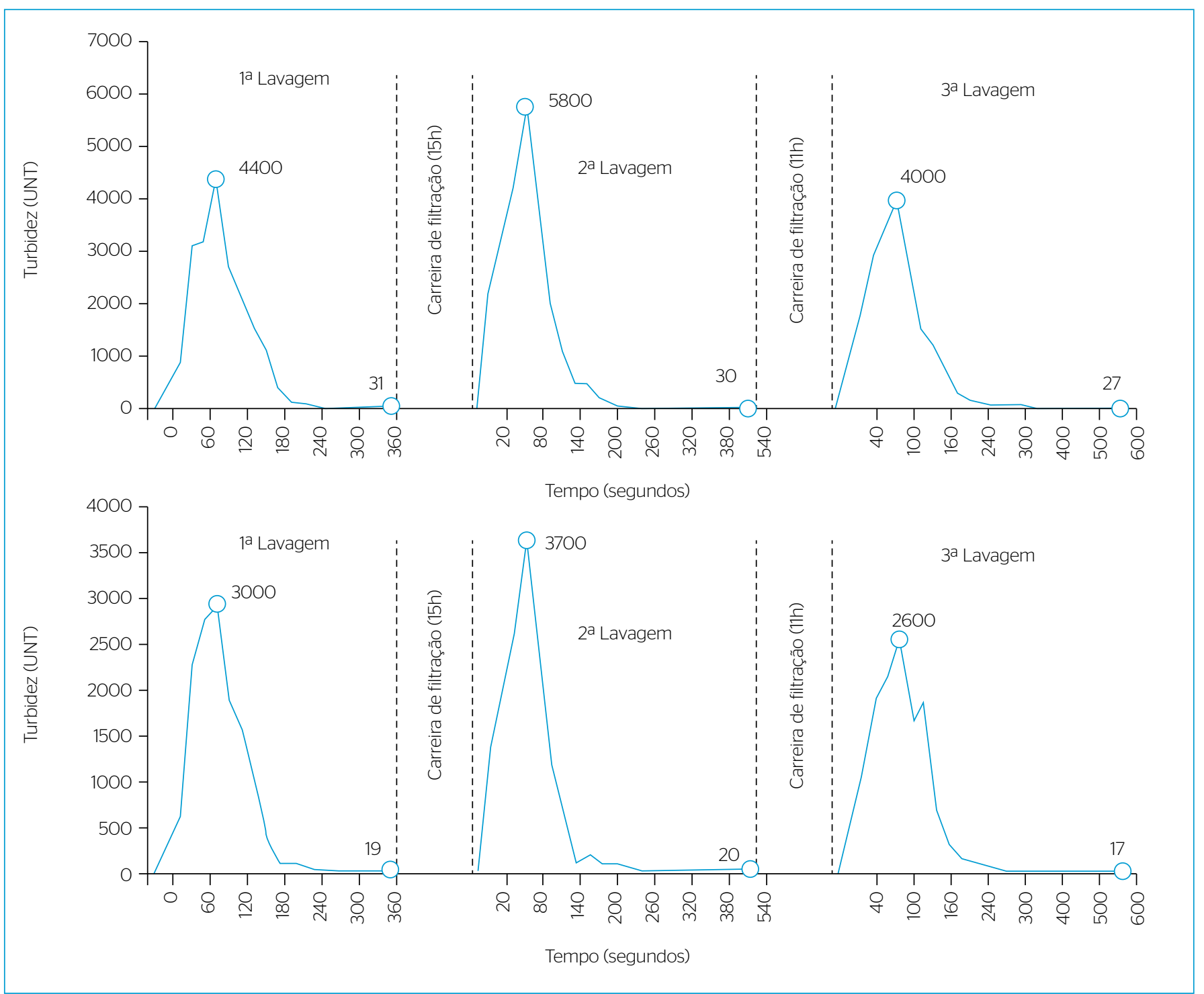

Figura 4 - Perfil das concentrações de turbidez e sólidos totais nos efluentes das lavagens 1, 2 e 3 do filtro 4. 
Ainda é oportuno ressaltar a presença de correlação entre turbidez e SST no efluente das três lavagens monitoradas. Essa correlação é representada pelo elevado valor de $\mathrm{R}^{2}$, que foi de 0,9715 .

Para um total de 70 pares de valores de turbidez e SST analisados nesta pesquisa, o coeficiente de correlação de Pearson $(r)$ resultou em 0,9857 . Assim, por essa análise estatística, há menos de $5 \%$ de chance $(\alpha=0,05)$ de que, sem correlação linear alguma, o valor de $r$ exceda o valor crítico de Pearson, que é de 0,236, atestando, dessa forma, a significância da correlação linear entre os parâmetros turbidez e SST para o efluente das lavagens do filtro 4 da unidade de tratamento em estudo. O elevado valor do $\mathrm{R}^{2}$, por sua vez, significa que $97,15 \%$ da variabilidade nos valores de turbidez encontrada neste estudo é explicada pelos valores de SST.

A correlação entre turbidez e SST tem sido significativa em grande parte dos estudos de qualidade da água, como pode ser observado nos trabalhos de Yao, Nan e Chen (2014) e Ribeiro et al. (2005). Em geral, a correlação varia com as características de cada tipo de água, podendo ser utilizada para se obter equações de regressão entre os parâmetros turbidez e SST. Esse recurso é importante para estudos sobre as condições ótimas de lavagem dos filtros que utilizam como parâmetro de análise a concentração de partículas. Tal característica pode ser observada nos estudos de Hall e Fitzpatrick (1998), Brouckaert et al. (2006) e Han, Fitzpatrick e Wetherill (2009), que usam as concentrações de partículas para o balanço de massa (entrada e saída de partículas do leito filtrante) em seus modelos propostos. Assim, pela regressão, a concentração de partículas no efluente de lavagem pode ser determinada indiretamente e de forma mais simples e barata, por meio da medição de turbidez. Com isso, a regressão é um recurso alternativo em potencial para simplificar e acelerar o estabelecimento das condições ótimas para a lavagem dos filtros.

Também é oportuno comentar a grande redução dos valores de turbidez na lavagem do filtro 4, que foi superior a 4000 UNT no início e passou para cerca de 30 UNT no final dos três períodos de lavagens do filtro monitorado. É interessante observar que o maior pico de turbidez ocorreu na segunda lavagem, o que, inicialmente, pode ser explicado pelo maior tempo na carreira de filtração anterior (15h) ter aumentado a retenção de partículas no leito filtrante. Contudo, diferentemente do esperado, o maior volume de água não ocorreu na segunda lavagem, mas sim na terceira lavagem do filtro, conforme pode ser observado na Figura 5.

Apesar dos grandes volumes de água utilizados (59, 65 e 67 L/s), a qualidade dos efluentes na primeira, segunda e terceira lavagens foi superior aos valores de turbidez recomendados por Cleasby e Logsdon (1999) e Kawamura (2000), indicando a necessidade de alterações operacionais e de adequação do leito filtrante, para melhorar a eficiência e padronizar a rotina de lavagem dos filtros da ETA UFPA.

Alterações operacionais na unidade de filtração da ETA UFPA, no entanto, são limitadas, pois o REL apresenta dupla função (lavagem do filtro e distribuição de água) e é utilizado na capacidade limite, o que gera grande risco de interrupção no abastecimento de água nos setores do campus nos momentos de grande demanda. Essa limitação dificulta a padronização da rotina e do tempo de lavagem, reforçando a importância de construção de sistema exclusivo para a lavagem adequada dos filtros.

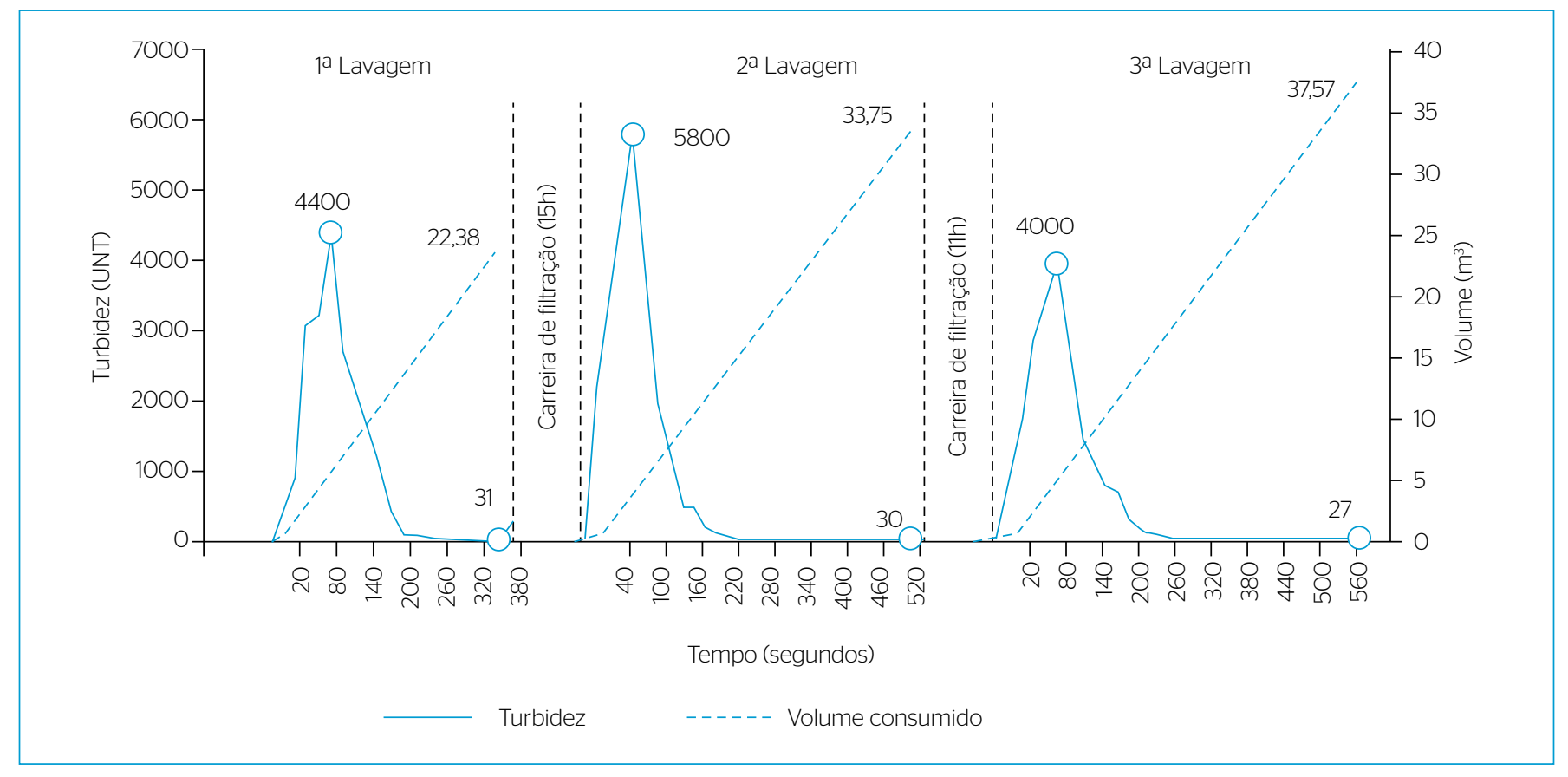

Figura 5 - Volume de água consumido ao longo da $1^{\mathrm{a}}, 2^{\mathrm{a}}$ e $3^{\mathrm{a}}$ lavagem do filtro 4. 
A Associação Brasileira de Normas Técnicas (1992) recomenda, em sua NBR 12.216/1992, um REL de lavagem da unidade de filtração dimensionado para tempo mínimo de 10 minutos de lavagem. Por sua vez, Richter (2009) cita que o REL deve ter dimensões mínimas que possibilitem tempo igual ou maior que 8 minutos de lavagem à máxima taxa.

Na pesquisa também foi determinado o $\mathrm{IPA}_{\mathrm{LF}}$ de $4,75 \%$ do volume total de água bruta, o que indica a necessidade de reavaliação do volume de água tratada utilizado na operação de lavagem do filtro, especialmente porque volume impactar no consumo e na despesa de energia elétrica no SAA da UFPA. Para possibilitar essa avaliação, na Tabela 2 são relacionados os valores dos monitoramentos hidroenergéticos realizados na EAB e na EAT.

$\mathrm{Na}$ rotina operacional monitorada foi determinado o valor de energia agregada de $0,52 \mathrm{kWh} / \mathrm{m}^{3}$ para deslocar o volume de água utilizado na lavagem de um filtro, sendo considerada a energia elétrica utilizada para impulsionar $1 \mathrm{~m}^{3}$ de água de $1125 \mathrm{kWh} /$ dia na EAB e de $280 \mathrm{kWh} /$ dia na EAT, o que resultou em CEEs de, respectivamente, 0,29 e $0,23 \mathrm{kWh} / \mathrm{m}^{3}$.

Como as estações de bombeamento apresentam características diferentes, na pesquisa foi calculado o CEEN, para possibilitar a comparação do desempenho hidroenergético dessas duas instalações de bombeamento. O menor valor do CEEN da EAB em relação ao da EAT demonstra que a EAB apresenta melhor desempenho

Tabela 2 - Dados do monitoramento hidroenergético na elevatória de água bruta e na elevatória de água tratada.

\begin{tabular}{|c|c|c|c|c|}
\hline Bombeamento & \multicolumn{2}{|c|}{ Indicadores de desempenho } & $\begin{array}{c}\text { CEE } \\
\left(\mathrm{kWh} / \mathrm{m}^{3}\right)\end{array}$ & $\begin{array}{l}\text { CEEN } \\
(\mathrm{kWh} /\end{array}$ \\
\hline \multirow{5}{*}{$E A B$} & $\mathrm{Hm}_{1}(\mathrm{~m})$ & 43,64 & \multirow{5}{*}{0,29} & \multirow{5}{*}{0,66} \\
\hline & $V_{1}\left(m^{3} / d i a\right)$ & $3.928,00$ & & \\
\hline & $\mathrm{E}_{1}(\mathrm{kWh} / \mathrm{dia})$ & 1125,00 & & \\
\hline & $\begin{array}{l}\text { Tempo de } \\
\text { funcionamento } \\
\text { diário (horas) }\end{array}$ & 21,71 & & \\
\hline & $\begin{array}{l}\% \text { de } \\
\text { funcionamento } \\
\text { diário }\end{array}$ & 90,43 & & \\
\hline \multirow{5}{*}{ EAT } & $\mathrm{Hm}_{2}(\mathrm{~m})$ & 26,31 & \multirow{5}{*}{0,23} & \multirow{5}{*}{0,86} \\
\hline & $V_{2}\left(m^{3} / d i a\right)$ & $1.234,00$ & & \\
\hline & $E_{2}(k W h / d i a)$ & 280,00 & & \\
\hline & $\begin{array}{l}\text { Tempo de } \\
\text { funcionamento } \\
\text { diário (horas) }\end{array}$ & 15,33 & & \\
\hline & $\begin{array}{l}\% \text { de } \\
\text { funcionamento } \\
\text { diário }\end{array}$ & 0,64 & & \\
\hline \multicolumn{2}{|c|}{ Total (CEE1+CEE2) } & & 0,52 & \\
\hline
\end{tabular}

CEE: consumo específico de energia; CEEN: consumo específico de energia normalizado; EAB: elevatória de água bruta; EAT: elevatória de água tratada hidroenergético, já que utiliza menor quantidade de energia elétrica para recalcar $1 \mathrm{~m}^{3}$ de água.

Para avaliar o resultado hidroenergético das três rotinas de lavagem do filtro, foram elaborados gráficos que relacionam os valores de turbidez com os de energia agregada, conforme pode ser observado na Figura 6.

Considerando-se o valor de turbidez da ordem de 30 UNT no final das 3 lavagens monitoradas, é possível observar que o melhor resultado em relação à energia agregada ocorreu na primeira lavagem $(12,32 \mathrm{kWh})$. Isso pode ser utilizado como indicativo de que as três lavagens poderiam ser finalizadas com 380 segundos (6,3 minutos), ou seja, para obter o mesmo valor de turbidez (da ordem de 30 UNT) foi utilizado volume desnecessário de água na segunda e terceira lavagens.

O consumo médio de energia elétrica por lavagem no filtro 4, considerando-se apenas o volume útil (até os 380 segundos de lavagem), foi de $12,15 \mathrm{kWh}$, e a extrapolação desse valor para os quatro filtros resulta no consumo de 97,2 kWh/dia na ETA, totalizando $2.916 \mathrm{kWh} / \mathrm{mês}$.

$\mathrm{Na}$ determinação da despesa com energia elétrica foi verificado que o SAA não apresenta medição independente de energia elétrica, isto é, a ETA faz parte da única unidade consumidora do campus universitário da UFPA, que apresenta sistema elétrico trifásico e é enquadrado na tarifa horo-sazonal verde, com classe de tensão A4 (2,3 a $25 \mathrm{kV})$.

Em relação à tarifação do consumo, foram verificados os valores de $\mathrm{R} \$ 2,06628 / \mathrm{kWh}$ no $\mathrm{P}$, que vai das $18 \mathrm{~h}$ às $20 \mathrm{~h} 59$, e de $\mathrm{R} \$ 0,26789 / \mathrm{kWh}$ no horário FP, sendo o preço da energia reativa excedente em P e FP de $\mathrm{R} \$ 0,21376 / \mathrm{kVArh}$ e o valor da demanda de $\mathrm{R} \$ 18,16286 / \mathrm{kW}$.

Para verificar a qualidade da energia elétrica, no monitoramento foram aquisitados dados de tensão (U), de corrente (I) e de potências ativa (em kW), reativa (em kWAr) e aparente (em kVA), para a determinação do fator de potência $(\operatorname{Cos} \varphi)$ na EAB (ponto E1) e na EAT (ponto E2), conforme pode ser observado na Tabela 3.

Como os fatores de potência indutivos nas 3 fases da instalação elétrica da EAB e da EAT foram inferiores a 0,92, verificou-se que a energia elétrica fornecida ao SAA da UFPA não é aproveitada adequadamente. Isso pode resultar na aplicação de multas previstas na Resolução Normativa $\mathrm{n}^{\circ} 414$, de 9 de setembro de 2010, da ANEEL. De acordo com Brasil (2010), fatores de potências baixos oneram a despesa com $\mathrm{m}^{3}$ produzido, pois o órgão regulador entende que a energia elétrica está sendo mal aproveitada pela unidade consumidora.

Com base no enquadramento tarifário, na operação da EAB e da EAT no P e no FP, bem como na qualidade da energia elétrica, foi utilizada a Equação 5 para determinar a despesa do $\mathrm{m}^{3}$ da água de lavagem da ETA da UFPA, cujo valor final foi de $1,36 \mathrm{R} \$ / \mathrm{m}^{3}$. É oportuno ressaltar que nesse valor não foram incluídos os tributos e descontos.

Com a determinação da despesa por $\mathrm{m}^{3}$, é possível observar na Figura 7, o aumento da despesa, durante as lavagens. 
O valor médio da despesa na lavagem do filtro 4, considerando-se os tempos de lavagem adotados nesta pesquisa, foi de $\mathrm{R} \$ 43,15$, ou seja, em todas as unidades de filtração da ETA é possível estimar o valor diário total de lavagem de 345,23 R \$/dia, o que resulta em despesas de $10.356,9$ R \$/mês e de R\$ 124.281,60/ano.

Como a partir de 380 segundos não existe variação significativa de turbidez e SST no efluente da lavagem, a finalização da lavagem nesse tempo reduzirá a despesa de energia elétrica para 31,83 $\mathrm{R} \$ /$ lavagem, resultando, para todos os filtros da ETA, em despesas de R\$254,64/ dia, R\$ 7.639,20/mês e R\$ 91.670,4/ano.

\section{CONCLUSÕES}

O efluente da lavagem do filtro 4 apresentou valores de turbidez e de SST superiores aos valores recomendados na literatura técnica. Esse fato pode ser explicado por rotina operacional, características do leito filtrante, tempo de lavagem e limitações do REL. O principal impacto negativo dessas características é que quanto maior o valor da turbidez do efluente no final da lavagem, menor a duração da carreira de filtração posterior. Por isso, é necessário melhorar o controle e relacionar o desempenho da carreira em função da lavagem anterior do filtro.

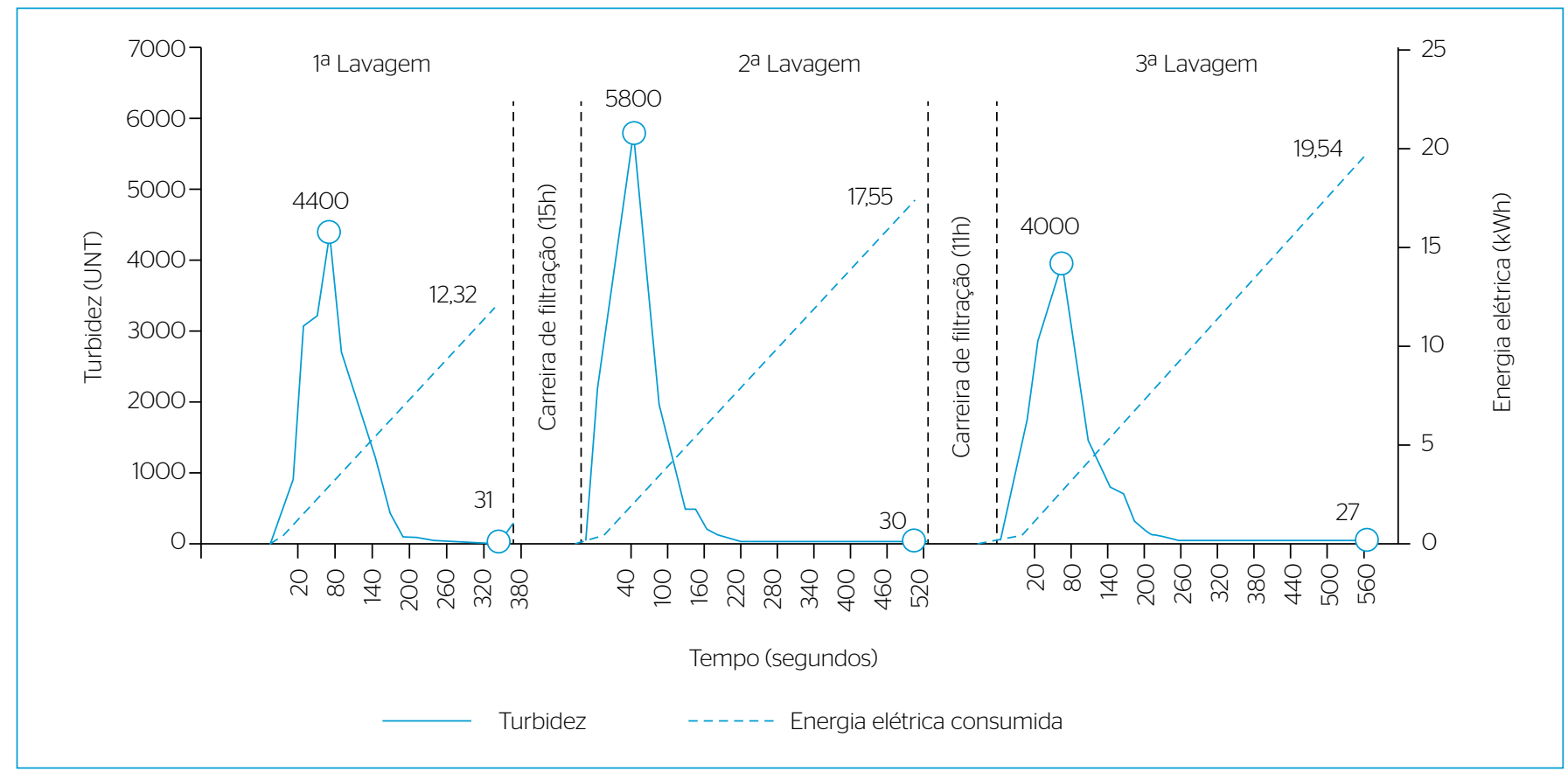

Figura 6 - Consumo de energia elétrica agregada ao longo das lavagens 1, 2 e 3.

Tabela 3 - Qualidade da energia elétrica consumida na elevatória de água bruta e na elevatória de água tratada.

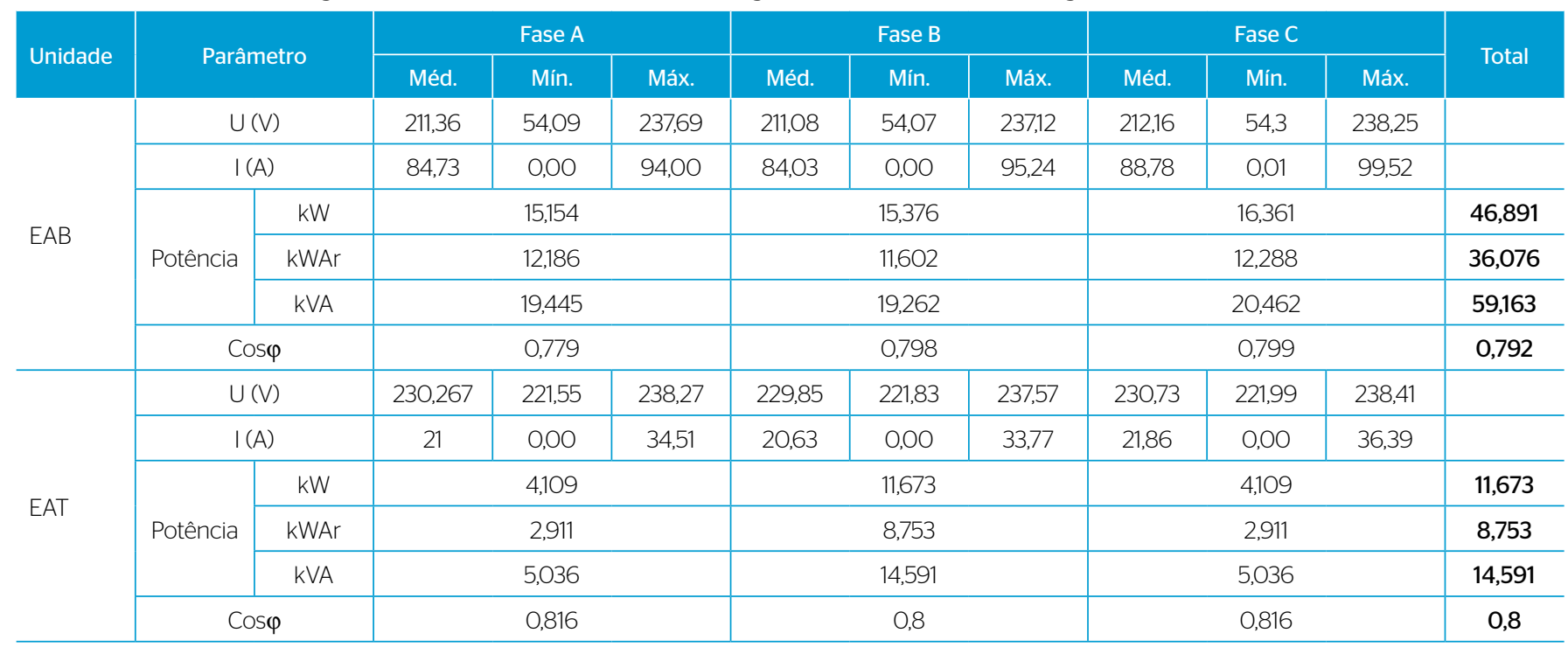

EAB: elevatória de água bruta; EAT: elevatória de água tratada. 


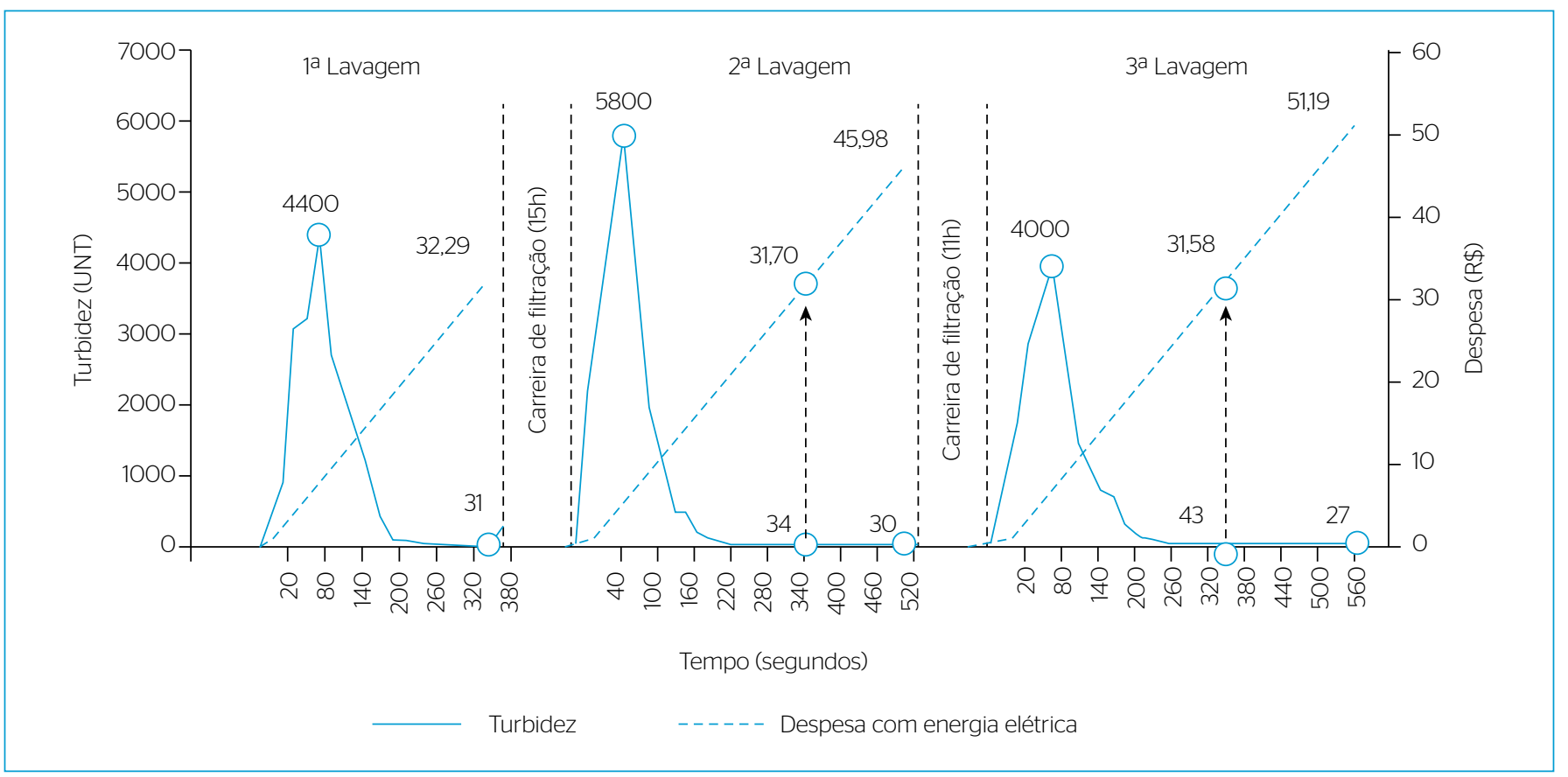

Figura 7 - Despesa dos volumes de água utilizados ao longo das lavagens 1, 2 e 3.

Atualmente, a dupla função do REL de água, no caso, lavagem de filtros e alimentação de parte do SAA do campus, impossibilita o armazenamento e a utilização de água bombeada no FP (água mais barata) nas lavagens. Com isso, para as lavagens noturnas e a continuidade do abastecimento pelo reservatório, é preciso bombeamento de água no $\mathrm{P}$ (água mais cara), resultando em lavagens com despesa de energia elétrica mais elevada do que o necessário.

Esse consumo de energia elétrica agregada à água de lavagem pode ser considerado elevado para a qualidade obtida no efluente dessas lavagens. Assim, além da diminuição do consumo da energia elétrica, faz-se necessária também a redução do custo, obtida pela elevação o $\operatorname{Cos} \varphi$ da EAB e da EAT. A elevação desse parâmetro pode ser obtida pela instalação de banco de capacitores. Além dessa medida, é essencial que sejam estabelecidas formas de evitar o bombeamento de água no $P$.
De forma emergencial, é preciso manter em 380 segundos as lavagens dos filtros, pois não são observadas reduções significativas de turbidez e SST após esse período.

O uso racional de água e de energia elétrica requer monitoramento constante, para que o controle eficiente possibilite a padronização de rotinas e as alterações apontadas no presente trabalho.

\section{AGRADECIMENTOS}

Os autores agradecem ao Conselho Nacional de Desenvolvimento Científico e Tecnológico $(\mathrm{CNPq})$ e à Coordenação de Aperfeiçoamento de Pessoal de Nível Superior (CAPES), pelas bolsas concedidas.

\section{REFERÊNCIAS}

ALEGRE, H.; HIRNER, W.; BAPTISTA, J.M.; PARENA, R. (2004) Indicadores de desempenho para serviços de abastecimento de água. Lisboa: IWA, Laboratório Nacional de Engenharia Civil, Instituto Regulador de Águas e Resíduos.

ASSOCIAÇÃO BRASILEIRA DE NORMAS TÉCNICAS (ABNT). (1992) NBR 12.216: projeto de estação de tratamento de água para abastecimento público. Rio de Janeiro: ABNT.

BRASIL. (2004) Ministério das Cidades. Secretaria Nacional de Saneamento Ambiental. Programa Nacional de Combateao Desperdício de Água: documento técnico de apoio: DTA A2: indicadores de perdas nos sistemas de abastecimento de água. Brasília: Ministério das Cidades.
BRASIL. (2010) Agência Nacional de Energia Elétrica (ANEEL). Resolução Normativa no 414, de 9 de setembro de 2010. Estabelece as Condições Gerais de Fornecimento de Energia Elétrica de forma atualizada e consolidada. Disponível em: <http:// www.aneel.gov.br/documents/656877/14486448/bren2010414 pdf/3bd33297-26f9-4ddf-94c3-fO1d76d6f14a?version=1.0>. Acesso em: 27 nov. 2016.

(2015) Sistema Nacional de Informações Sobre Saneamento (SNIS). Diagnóstico dos Serviços de Água e Esgoto-2013. Brasília. Disponível em: <http://www.snis.gov.br/ diagnostico-agua-e-esgotos/diagnostico-ae-2013>. Acesso em: 4 ago. 2018. 
(2015) Agência Nacional de Energia Elétrica (ANEEL). Resolução homologatória no 1.859, de 27 de fevereiro de 2015. Estabelece as faixas de acionamento e os adicionais das bandeiras tarifárias, de que trata o submódulo 6.8 do PRORET, com vigência a partir de 2 de março de 2015. Disponível em: <http:// www2.aneel.gov.br/arquivos/PDF/Resolucao_Homologatoria. pdf>. Acesso em: 14 jan. 2020

(2016) Sistema Nacional de Informações Sobre Saneamento (SNIS). Diagnóstico dos Serviços de Água e Esgoto-2014. Brasília. Disponível em: <http://www.snis.gov.br/diagnostico-agua-eesgotos/diagnostico-ae-2014>. Acesso em: 4 ago. 2018.

(2017) Sistema Nacional de Informações Sobre Saneamento (SNIS). Diagnóstico dos Serviços de Água e Esgoto-2015. Brasília. Disponível em: <http://www.snis.gov.br/diagnostico-agua-eesgotos/diagnostico-ae-2015>. Acesso em: 4 ago. 2018.

(2018) Sistema Nacional de Informações Sobre Saneamento (SNIS). Diagnóstico dos Serviços de Água e Esgoto-2016. Brasília. Disponível em: <http://www.snis.gov.br/diagnostico-agua-eesgotos/diagnostico-ae-2016>. Acesso em: 4 ago. 2018.

BROUCKAERT, B.M.; AMIRTHARAJAH, A.; BROUCKAERT, C.J.; AMBURGEY, J.E. (2006) Predicting the efficiency of deposit removal during filter backwash. Water S.A., v. 32, n. 5, p. 633-640. http://doi.org/10.4314/wsa.v32i5.47842

CHEUNG, P.B.; REIS, L.F.R., FORMIGA, K.T.M.; CHAUDHRY, F.H.; TICONA, W.G.C. (2003) Multiobjective Evolutionary Algorithms Applied to the Rehabilitation of a Water Distribution System: A Comparative Study. Lecture Notes in Computer Science, v. 2632, p. 662-676. https://doi.org/10.1007/3-540-36970-8_47

CLEASBY, J.L.; LOGSDON, G.S.L. (1999) Granular bed and precoat filtration. In: LETTERMAN, R.D. (org.) Water Quality and Treatment. 5. ed. Nova York: AWWA/McGraw-Hill.

FIRMINO, M.B.M.; ALBUQUERQUE, A.A. de; CURI, W.F.; SILVA, N.C. da. (2006) Método de Eficiência Energética no Bombeamento de Água via Programação Linear e Inteira. In: VI SEMINÁRIO IBEROAMERICANO SOBRE SISTEMAS DE ABASTECIMENTO URBANO DE ÁGUA, 6., 2006. João Pessoa.
FREITAS, A.G.; BASTOS, R.K.X.; BEVILACQUA, P.D.; PÁDUA, V.L.; PIMENTA, J.F.P.; ANDRADE, R.C. (2010) Recirculação de água de lavagem de filtros e perigos associados a protozoários. Engenharia Sanitária e Ambiental, v. 15, n. 1, p. 37-46. http://dx.doi.org/10.1590/ S1413-41522010000100005

GOMES, H.P. (2009) Sistemas de bombeamento: Eficiência energética. João Pessoa: Ed. UFPB. 460 p.

GUMIER, C.C.; LUVIZOTTO JUNIOR, E. (2007) Aplicação do Modelo de Simulação - Otimização na Gestão de Perda de Água em Sistemas de Abastecimento. Engenharia Sanitária e Ambiental, v. 12, n. 1, p. 32-41. http://dx.doi.org/10.1590/S1413-41522007000100005

HALL, D.; FITZPATRICK, C.S.B. (1998) A mathematical filter backwash Model. Water Science and Technology, v. 37, n. 12, p. 371-379. https:// doi.org/10.1016/SO273-1223(98)00358-8

HAN, S.J.; FITZPATRICK, C.S.B.; WETHERILL, A. (2009) Simulation on combined rapid gravity filtration and backwash models. Water Science \& Technology, v. 59, n. 12, p. 2.429-2.435. https://doi. org/10.2166/wst.2009.308

KAWAMURA, S. (2000) Integrated Design and Operation of Water Treatment Facilities. 2. ed. Nova York: Wiley.

PEREIRA, J.A.; CONDURÚ, M.T. (2014) Abastecimento de água: informação para eficiência hidroenergética. João Pessoa: Ed. UFPB. 127 p.

RIBEIRO, T.A.R.; AIROLDI, R.P. da S.; PATERNIANI, J.E.S.; SILVA, M.J.M. (2005) Variação dos parâmetros físicos, químicos e biológicos da água em um sistema de irrigação localizada. Revista Brasileira de Engenharia Agrícola e Ambiental, v. 9, n. 3, p. 295-301. http://dx.doi. org/10.1590/S1415-43662005000300001

RICHTER, C.A. (2009) Água: métodos e tecnologia de tratamento. São Paulo: Blucher. 340 p.

YAO, M.; NAN, J.; CHEN, T. (2014) Effect of particle size distribution on turbidity under various water quality levels during flocculation processes. Desalination, v. 354, p. 116-124. https://doi.org/10.1016/j. desal.2014.09.029 